\title{
Disease Self-Efficacy and Health-Related Quality of Life in Adolescents with Sickle Cell Disease
}

\author{
Alana Goldstein-Leever, Psy.D ${ }^{1,2}$, James L. Peugh, Ph.D ${ }^{3,4}$, Charles T. Quinn, M.D., M.S ${ }^{4,5}$, \\ and Lori E. Crosby, Psy. $\mathbf{D}^{3,4}$ \\ 1Division of Pediatric Psychology and Neuropsychology, Nationwide Children's Hospital, \\ Columbus, $\mathrm{OH}$ \\ 2Department of Pediatrics, The Ohio State University, Columbus, $\mathrm{OH}$ \\ ${ }^{3}$ Division of Behavioral Medicine, Cincinnati Children's Hospital Medical Center, Cincinnati, $\mathrm{OH}$ \\ ${ }^{4}$ Department of Pediatrics, University of Cincinnati College of Medicine, Cincinnati, $\mathrm{OH}$ \\ ${ }^{5}$ Division of Hematology, Cincinnati Children's Hospital Medical Center, Cincinnati, $\mathrm{OH}$
}

\begin{abstract}
Adolescents and young adults (AYA) with sickle cell disease (SCD) are at risk for poor healthrelated quality of life (HRQOL). Research suggests that vulnerability factors (e.g., disease severity) and self-management resources (e.g., disease self-efficacy) jointly impact health outcomes, including HRQOL; however, this has not been studied among AYA with SCD. This study examined the relationship between disease self-efficacy, HRQOL, and disease severity in AYA with SCD. HRQOL was positively correlated with disease self-efficacy and negatively correlated with disease severity. Disease self-efficacy and severity accounted for $35 \%$ of variance in HRQOL. Findings support the impact of disease self-efficacy on HRQOL.
\end{abstract}

\section{Keywords}

health-related quality of life; disease self-efficacy; adolescents and young adults; sickle cell disease.

\section{Introduction}

Adolescents and young adults (AYA) with sickle cell disease (SCD) are at risk for poor health outcomes, including impaired health-related quality of life (HRQOL). SCD is a chronic, heritable blood disorder affecting 70,000 to 100,000 individuals in the United States, primarily those of African American or Hispanic descent [1]. SCD is associated with significant healthcare utilization and lifelong disease complications, including chronic

Correspondence concerning this paper should be addressed to: Alana Goldstein-Leever, Psy.D., Clinical Assistant Professor of Pediatrics; The Ohio State University, Nationwide Children's Hospital; Address: 700 Children's Drive Columbus, Ohio $43205-2664$. Phone: (614) 722-4700, Fax: (614) 722-4718, alana.leever@nationwidechildrens.org.

Conflicts of Interest and Source of Funding

The authors have no conflicts of interest to declare. 
anemia, recurrent vaso-occlusive events, and ongoing organ damage [2]. Adolescence and young adulthood represents a particularly vulnerable time in the SCD trajectory, as the toll of cumulative organ damage throughout childhood manifests in higher rates of acute care utilization, morbidity, and early mortality in AYA than the younger SCD population [2-4].

Jenerette and Murdaugh's (2008) theory of self-care management suggests that vulnerability factors (e.g., disease severity) and modifiable self-management resources (e.g., disease selfefficacy) jointly impact health outcomes in the SCD population [5]. AYA with SCD, in particular, face significant shifts in healthcare provisions, their support network, and expectations for self-management, which affect their functioning across domains. These lapses in healthcare access and supportive programming, insurance coverage, and disease self-management skills are compounded by AYAs' emerging risk for medical complications, neurocognitive sequelae, and adjustment difficulties [6]. Normative shifts in the developmental tasks of adolescence pose an added challenge for AYA with SCD, as they are expected to make progress towards educational, vocational, and social goals, while reconciling increasing demands for disease self-management and independent functioning [7]. Despite this, AYA with SCD demonstrate low rates of medical adherence and insufficient disease self-management skills, likely influenced by their underlying perceptions of disease self-efficacy [8-9].

Disease self-efficacy refers to one's personal appraisal of his/her capacity to manage disease care, promote symptom management, and support appropriate functioning in the context of chronic medical needs [10-11]. It has been associated with better health outcomes among AYA with chronic medical conditions, including sickle cell disease [5,11-13]. Among AYA with SCD, disease self-efficacy has been linked to improved physical and psychological health [10-12,14], and increased readiness for transition [13-14]. Furthermore, literature about adults with SCD supports the relationship between disease self-efficacy and HRQOL [15]. However, to our knowledge, no studies to date have directly explored the relationship between disease self-efficacy and HRQOL in AYA with SCD.

Considering the medical, psychological, and socioecological factors affecting this group, AYA with SCD represent a vulnerable population in need of timely intervention to improve disease self-management and HRQOL. Understanding the impact of disease self-efficacy on HRQOL represents a critical first step towards designing health behavior interventions that may facilitate positive health outcomes. Given that disease severity may independently impact HRQOL, this study examined the relationship between disease self-efficacy, HRQOL, and disease severity in AYA with SCD. We hypothesized that disease self-efficacy would significantly predict HRQOL among AYA with SCD [5]. AYA with overtly mild SCD and/or increased disease self-efficacy were expected to demonstrate better HRQOL than those with overtly severe SCD and/or lower levels of disease self-efficacy $[5,11,12-13,16]$.

\section{Materials and Methods}

\section{Participants}

AYA were eligible ( $\mathrm{N}=101)$ if they had SCD, were between the ages of 13 and 22 (an age range informed by guiding literature on AYA in SCD [9]), and received care from a 
multidisciplinary SCD clinic at an urban pediatric medical center. AYA were recruited during clinic appointments or community outreach events. Although this was a clinical outcomes study and the data was used to inform about standard clinical care, institutional review board approval was also obtained.

\section{Measures}

Family Information Form.-Adolescents' caregivers or adult participants ( $\geq 18$ ) completed a sociodemographic questionnaire adapted from the Pediatric Quality of LifeFamily Information Form [17].

Pediatric Quality of Life, Sickle Cell Disease Module, Self-Report(PedsQL ${ }^{T_{M}}$ SCD).-The PedsQL ${ }^{\mathrm{TM}}-\mathrm{SCD}$ is a 43 -item, reliable and valid measure of disease-specific HRQOL that has demonstrated excellent internal consistency in the current sample (Cronbach's $a=.97$ ) [16]. Total scores were calculated, with higher scores suggesting improved HRQOL.

Sickle Cell Self-Efficacy Scale (SCSES).-The SCSES is a 9-item, self-report measure of disease self-efficacy that was designed for use in the SCD population [1112,13]. The SCSES has proven reliable and valid among AYA with SCD and demonstrated good internal consistency within the current sample (Cronbach's $a=.80$ ) [12]. Total scores were calculated, with higher scores suggesting improved disease self-efficacy.

Disease Severity.-Disease severity was dichotomized into mild $(n=34)$ and severe $(n=$ 45) classifications. Participants with severe disease had a history of overt stroke, acute chest syndrome, or three or more admissions for vaso-occlusive pain within the preceding threeyear period, based on medical record report [16].

\section{Data Analytic Plan}

Statistical analyses were performed using SPSS, Version 23, including multiple imputation to address missing data. Descriptive statistics summarized demographic, medical, and psychosocial characteristics. Pearson's correlations explored the relationships among primary variables of interest. Hierarchical regression analyses examined the predictive value of disease self-efficacy and disease severity on HRQOL.

\section{Results}

\section{Sample Characteristics}

Of 101 AYA screened for eligibility, 79 participants (13-22 years; $M=15.9, S D=2.4$ ) enrolled and completed measures. In general, participants had the HbSS genotype of SCD, were African American, and publically insured; gender distribution was approximately equal. See Table 1 for full demographic data and Table 2 for means and standard deviations of all primary outcome variables. Of 22 AYA excluded from the study, 18 declined participation and 4 did not meet inclusion criteria due to documented cognitive disability (n $=2$ ) or based on the recommendation of their healthcare provider $(n=2)$. Patients who declined participation $(n=18)$ were demographically and medically comparable to those 
included in the study $\left(M_{\mathrm{age}}=16.7, S D_{\mathrm{age}}=2.4\right.$; African American $100 \%$; publicly insured $72 \%$; and had the HbSS genotype 61\%).

\section{Disease Self-Efficacy, HRQOL and Disease Severity}

Disease self-efficacy was positively related to HRQOL $(r=.536, p<.001)$ and negatively related to disease severity $(r=-.304, p<.01)$. Disease severity was also negatively related to HRQOL $(r=-.304, p<.01)$. The final regression model, including disease self-efficacy and disease severity, accounted for $35 \%$ of variance in HRQOL, $F \Delta(1,76)=30.10, p<$. 001.B See Table 3 for a full summary of hierarchical regression analyses. Both disease selfefficacy $(\mathrm{B}=1.72, \mathrm{SE}=0.32, p<.001)$ and disease severity $(\mathrm{B}=-.11 .54, \mathrm{SE}=4.13, p<.01)$ were statistically significant predictors of HRQOL.

\section{Discussion}

This study examined the relationship between disease self-efficacy, HRQOL, and disease severity in a sample of AYA with SCD. Consistent with our hypotheses [5,11-13,16], we found that disease self-efficacy significantly predicted HRQOL and those with mild SCD or increased disease self-efficacy demonstrated better HRQOL. Given trends of declining health and adjustment among AYA with SCD, our results inform research and clinical efforts to improve health outcomes in this population [1-2,6]. Jenerette and Murdaugh's (2008) framework conceptualizes disease self-efficacy as a modifiable factor, while disease severity may be a vulnerability factor and more challenging to address [5]. Interventions to promote disease self-efficacy and self-management skills may be especially beneficial given the potential impact of such programming on health outcomes. Multicomponent selfmanagement programs, for example, have been widely applied across chronic disease populations to support disease self-management, adherence, and healthy behaviors among youth and adults with chronic medical conditions [18]. One such program, the Stanford Chronic Disease Self-Management Program, has demonstrated initial feasibility and acceptability among AYA with SCD, resulting in improvements in global self-efficacy [19]. Further research is needed to develop and evaluate additional interventions, including disease-specific interventions, targeting disease self-efficacy and self-management in this high-risk population.

As expected, HRQOL was highest among AYA with mild SCD and those with higher levels of disease self-efficacy. Importantly, this relationship between disease self-efficacy and HRQOL was significant, even when controlling for disease severity. Findings suggest that AYAs' perceived ability to cope with SCD, notwithstanding disease status, significantly contributes to HRQOL. This is consistent with extant literature among AYA with SCD, suggesting that adolescents' perceptions of their illness directly inform HRQOL [20]. Further research is presently needed to explore the clinical utility of disease self-efficacy assessment, including the development of clinical cut-off scores for the SCSES. Such data may be used to inform score interpretation and clinical decision-making, guiding the process of identifying AYA with limited disease self-efficacy who might benefit from intervention.

Findings must be interpreted within the context of study limitations, which included use of a relatively small, convenience sample at a single site. Furthermore, utilization of a cross- 
sectional study design negates the ability to make causal attributions. To truly understand the complex relationship between disease self-efficacy, HRQOL, and disease severity in in AYA with SCD, longitudinal studies are needed. Future studies should also examine the influence of disease self-efficacy interventions on HRQOL and other health outcomes. It remains important to note that a number of medical and psychosocial factors have been excluded from the current analysis, despite their potential impact on disease self-efficacy and HRQOL, including the presence of underlying mental health disorders, neurocognitive sequela of SCD (beyond overt stroke), and other medical/behavioral variables, such as fatigue or sleep disturbance. While the inclusion of psychosocial, cognitive, and sociodemographic variables was beyond the scope of this pilot study, these factors warrant further exploration and remain relevant considerations in the evaluation of health outcomes among AYA with SCD $[10,20]$. Importantly, disease severity was captured and controlled for across study analyses; however, dichotomization of this variable into mild and severe classifications (16) may serve to limit observed variability in patients' disease profiles and should be recognized as a potential limitation.

Given the compromised health and psychosocial outcomes among AYA with SCD, particularly in the context of healthcare transition, research is currently needed to aid in identifying factors that may influence HRQOL in this population. The current study provides preliminary data suggesting that disease self-efficacy uniquely predicts HRQOL, beyond the effects of medical characteristics like disease severity. In fact, disease selfefficacy may be best conceptualized as a modifiable risk factor for reduced HRQOL, with the potential to be addressed with intervention directed at disease self-management skills and mastery [19]. Results of the current study contribute to the available literature on HRQOL in AYA with SCD and provide future directions for clinical intervention and research.

\section{Acknowledgements}

The authors would like to acknowledge the patients and families for their time and participation in this research.

This work was supported in part by the National Heart, Lung, and Blood Institute at the National Institutes of Health (Grant \#: K07HL108720). Its contents are solely the responsibility of the authors and do not necessarily represent the official views of the National Institutes of Health.

\section{References}

1. Hassell KL. Population estimates of sickle cell disease in the U.S. Am J Prev Med. 2010;38:S512S521 [PubMed: 20331952]

2. Quinn CT. Sickle cell disease in childhood: From newborn screening through transition to adult medical care. Pediatr Clin North Am. 2013;60:1363-1381. [PubMed: 24237976]

3. Brousseau DC, Ownes PL, Mosso AL, et al. Acute care utilization and rehospitalizations for sickle cell disease. JAMA. 2010;303:1288-1294. [PubMed: 20371788]

4. Platt OS, Brambilla DJ, Rosse WF, et al. Mortality in sickle cell disease: Life expectancy and risk factors for early death. New Engl J Med. 1994;330:1639-1644. [PubMed: 7993409]

5. Jenerette CM, Murdaugh C. Testing the theory of self-care management for sickle cell disease. Res Nurs Health. 2008;31:355-369. [PubMed: 18247376]

6. Jordan L, Swerdlow P, Coates TD. Systematic review of transition from adolescent to adult care in patients with sickle cell disease. J Pediatr Hematol Oncol. 2013;35:165-169. [PubMed: 23511487] 
7. Baskin ML, Collins MH, Brown F, et al. Psychosocial considerations in sickle cell disease (SCD): The transition from adolescence to young adulthood. J Clin Psychol Med Settings. 1998;5:315-34.

8. Loiselle K, Lee JL, Szulczewski L, et al. Systematic and meta-analytic review: Medication adherence among pediatric patients with sickle cell disease. J Pediatr Psychol. 2016; 41:406-418. [PubMed: 26384715]

9. Mulchan SS, Valenzuela JM, Crosby LE, et al. Applicability of the SMART model of transition readiness for sickle-cell disease. J Pediatr Psychol. 2016;41:543-554. [PubMed: 26717957]

10. Edwards R, Telfair J, Cecil H, et al. Reliability and validity of a self-efficacy instrument specific to sickle cell disease. Behaviour Res Ther. 2000;38:951-963.

11. Edwards R, Telfair J, Cecil H, et al. Self-efficacy as a predictor of adult adjustment to sickle cell disease: One-year outcomes. Psychosom Med. 2001;63:850-858. [PubMed: 11573035]

12. Clay OJ, Telfair J. Evaluation of a disease-specific self-efficacy instrument in adolescents with sickle cell disease and its relationship to adjustment. Child Neuropsychol. 2007;13:188-203. [PubMed: 17364574]

13. Treadwell M, Johnson S, Bitsko M, et al. Self-efficacy and readiness for transition from pediatric to adult care in sickle cell disease. Int J Adolesc Med Health. 2016;28:381-388. [PubMed: 26226116]

14. Molter BL, Abrahamson K. Self-efficacy, transition, and patient outcomes in the sickle cell disease population. Pain Manag Nurs. 2015;16:418-424. [PubMed: 25047808]

15. Spirituality Adegbola M., self-efficacy, and quality of life among adults with sickle cell disease. South Online J Nurs Res. 2011;11:1-16.

16. Panepinto JA, Torres S, Bendo CB, et al. PedsQL ${ }^{\mathrm{TM}}$ sickle cell disease module: feasibility, reliability, and validity. Pediatr Blood Cancer 2013;60:1338-1344. [PubMed: 23441057]

17. Varni JW, Seid M, Kurtin PS. PedsQLTM 4.0: Reliability and validity of the Pediatric Quality of Life InventoryTM Version 4.0 Generic Core Scales in health and patient populations. Med Care. 2001;39:800-812. [PubMed: 11468499]

18. Modi AC, Pai AL, Hommel KA, et al. Pediatric self-management: A framework for research, practice, and policy. Pediatr 2012;129:e473-485.

19. Crosby LE, Joffe NE, Peugh J, et al. Pilot of the chronic disease self-management program for adolescents and young adults with sickle cell disease. J Adolesc Health 2017;60:120-123. [PubMed: 27793727]

20. Asnani MR, Barton-Gooden A, Grindley M, et al. Disease knowledge, illness perceptions, and quality of life in adolescents with sickle cell disease: Is there a link? Glob Pediatr Health 2017;4:1-10. 


\section{Table 1}

Demographic and Disease Characteristics of AYA with SCD $(\mathrm{N}=79)$

\begin{tabular}{|c|c|c|}
\hline & \multicolumn{2}{|c|}{$M(S D)$} \\
\hline Age & \multicolumn{2}{|c|}{$15.9(2.4)$} \\
\hline Annual Hospitalizations & \multicolumn{2}{|c|}{$0.92(1.92)$} \\
\hline & \multicolumn{2}{|c|}{$N(\%)$} \\
\hline \multicolumn{3}{|l|}{ Gender } \\
\hline Female & 38 & $(48.1)$ \\
\hline Male & 41 & $(51.9)$ \\
\hline \multicolumn{3}{|l|}{ Race/Ethnicity } \\
\hline African American & 79 & $(100.0)$ \\
\hline \multicolumn{3}{|l|}{ Highest Education $^{a}$} \\
\hline Grades 7-8 & 21 & $(29.2)$ \\
\hline Grades $9-12$ & 39 & $(54.2)$ \\
\hline High School Graduate & 3 & $(4.1)$ \\
\hline Some College/College Graduate & 9 & $(12.5)$ \\
\hline \multicolumn{3}{|l|}{ Insurance } \\
\hline Public (Medicaid) & 54 & $(68.4)$ \\
\hline Private & 22 & $(27.8)$ \\
\hline Multiple & 3 & $(3.8)$ \\
\hline \multicolumn{3}{|l|}{ Disease Severity } \\
\hline Mild & 34 & $(43.0)$ \\
\hline Severe & 45 & $(57.0)$ \\
\hline \multicolumn{3}{|l|}{ SCD Genotype } \\
\hline $\mathrm{HbSS}$ & 54 & $(68.4)$ \\
\hline $\mathrm{HbSC}$ & 19 & $(24.1)$ \\
\hline HbS $\beta$ thal & 5 & (6.3) \\
\hline $\mathrm{HbSD}$ & 1 & (1.3) \\
\hline
\end{tabular}

Note. Information was obtained from the electronic medical record or via patient/caregiver report on the Family Information Form. Mild=Mild $\mathrm{SCD}$; Severe=Severe SCD.

Includes select missing data $(n=72)$. 
Table 2

Descriptive Statistics for the SCSES and PedsQL-SCD Module

\begin{tabular}{lcc}
\hline Scale/Subscale & M & SD \\
\hline SCSES & 34.56 & 5.55 \\
PedsQL-SCD Total & 64.38 & 18.90 \\
$\quad$ Pain and Hurt & 65.26 & 22.50 \\
Pain Impact & 57.99 & 23.86 \\
Pain Management and Control & 65.82 & 24.08 \\
Worry I & 65.76 & 24.03 \\
Worry II & 75.47 & 23.47 \\
Emotions & 65.19 & 29.80 \\
Treatment & 66.05 & 20.91 \\
Communications I & 74.68 & 21.49 \\
Communications II & 57.81 & 26.30 \\
\hline
\end{tabular}

Note. Includes means $(M)$ and standard deviations $(S D)$ for primary outcome measures.

SCSES=Sickle Cell Self-Efficacy Scale, PedsQL-SCD=Pediatric Quality of Life-SCD Module 
Table 3

Disease severity and disease self-efficacy predict health-related quality of $\operatorname{life}(N=79)$

\begin{tabular}{|c|c|c|c|c|c|}
\hline Step/Predictor & B & SE & $R^{2}$ & $\Delta \mathbf{F}$ & $p$ \\
\hline Step 1 & & & .09 & 7.93 & .01 \\
\hline Disease Severity ${ }^{*}$ & -11.54 & 4.13 & & & \\
\hline Step 2 & & & .35 & 30.10 & .000 \\
\hline Disease Self-Efficacy ${ }^{* *}$ & 1.72 & 0.32 & & & \\
\hline
\end{tabular}

Note. Results of hierarchical regression analyses using multiple imputation to estimate pooled means.

$p<.05$

*** $p<.001$ 